\title{
Supplementary
}

\section{Aerosol delivery of paclitaxel containing self-assembled nanocochleates for treating pulmonary metastasis: an approach supporting pulmonary mechanics}

Thanigaivel Shanmugam $^{1}$, Nitin Joshi', Anubhav Kaviratna1, Nadim Ahamad', Eshant Bhatia and Rinti Banerjee ${ }^{1 *}$

1. Nanomedicine Laboratory, Department of Biosciences and Bioengineering, Indian Institute of Technology-Bombay, Mumbai-400076, India,

*Corresponding author:

Prof. Rinti Banerjee,

Nanomedicine Laboratory

Department of Biosciences and Bioengineering,

Indian Institute of Technology-Bombay,

Mumbai-400076, India

Email: rinti@,iitb.ac.in

Tel: + (91-22) 2576 7868;

Fax: + (91-22) 25723480 . 


\section{Fluoresce activated cell sorting (FACS) analysis of R6G-labelled CPT particles}

Figure S1 shows an overlay of dot plot of blank CPTs (cyan) and R6G labeled CPT (red) particles analyzed using FACS. The gating for the positive and negative population was done according to blank CPTs. The R6G-CPT population showed an evident right shift in fluorescence intensity due to the presence of R6G inside CPT particles. A total of $60.3 \%$ of R6G-CPT were fluorescence positive, confirming the successful retention of R6G dye in CPT particles even after rigorous washing steps involving high-speed centrifugation.
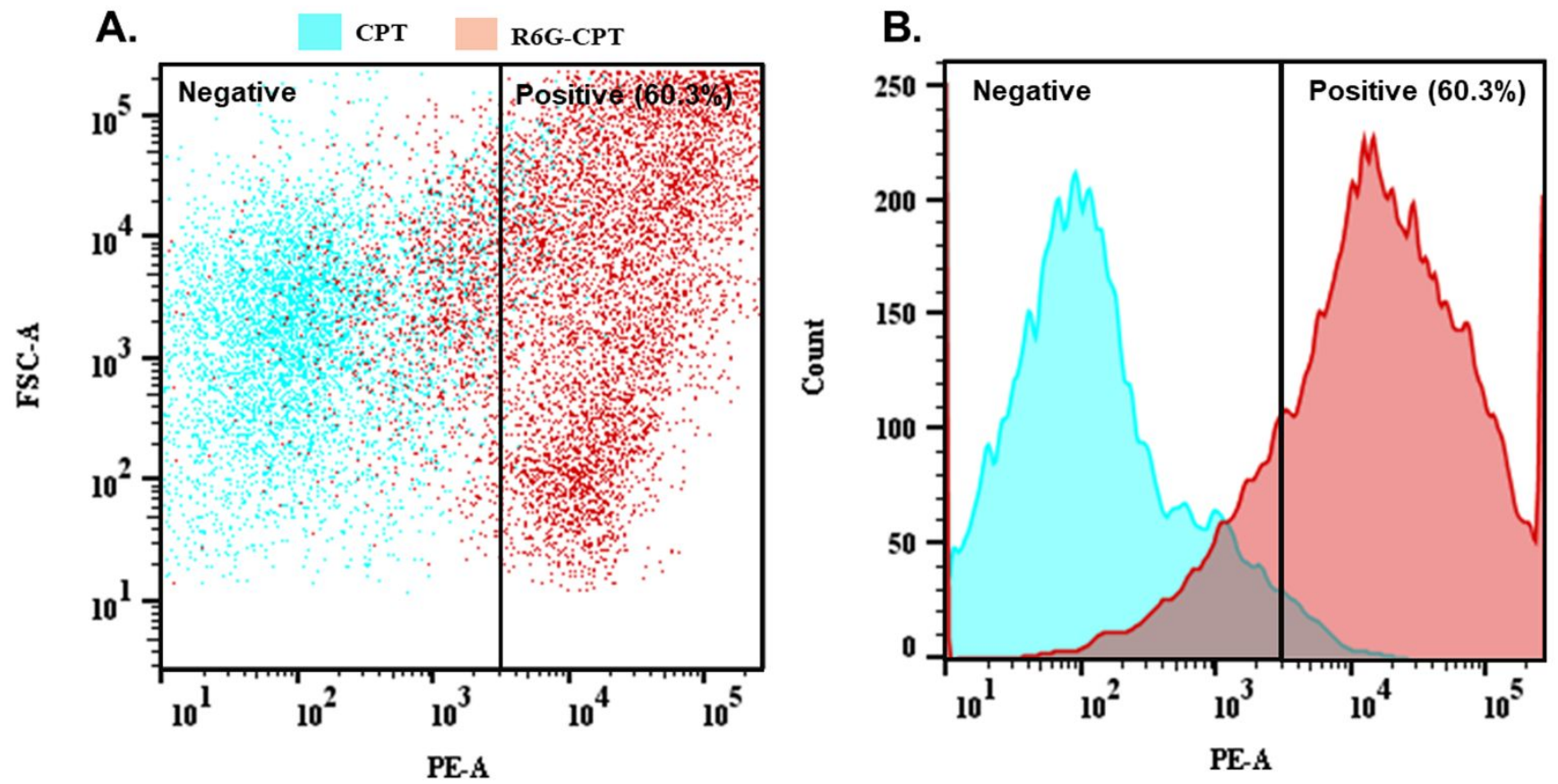

Figure S1 Representative FACS data of R6G-labelled CPT particles (without cells). A- Side scattering intensity distribution of singlet R6G-CPT species in the formulation, B-quantitation of singlet R6G negative (blank) CPT, and singlet R6G positive CPT particles.

\section{FACS analysis for CPT uptake by B16F10}

Figure S2 shows the representative fluorescence-shift due to R6G-CPT uptake by differently pre-treated B16F10 cells and following $1 \mathrm{~h}$ incubation (corresponding to figure 3C). A left-shift in population shows a decrease in fluorescence intensity suggesting the reduced uptake of R6G-CPT in cells pretreated for $1 \mathrm{~h}$ with $0.1 \%$ azide at $37^{\circ} \mathrm{C}$ and also at $4^{\circ} \mathrm{C}$. Reduced uptake of CPT particles in these cells 
suggested that these CPT particles were endocytosed by B16F10 cells involving an energy-dependent (active-uptake) endocytosis process as uptake of particles was predominantly inhibited in presence of metabolic inhibitor $(0.1 \%$ azide $)$ and even when the cells were pre-incubated at $4^{\circ} \mathrm{C}$.

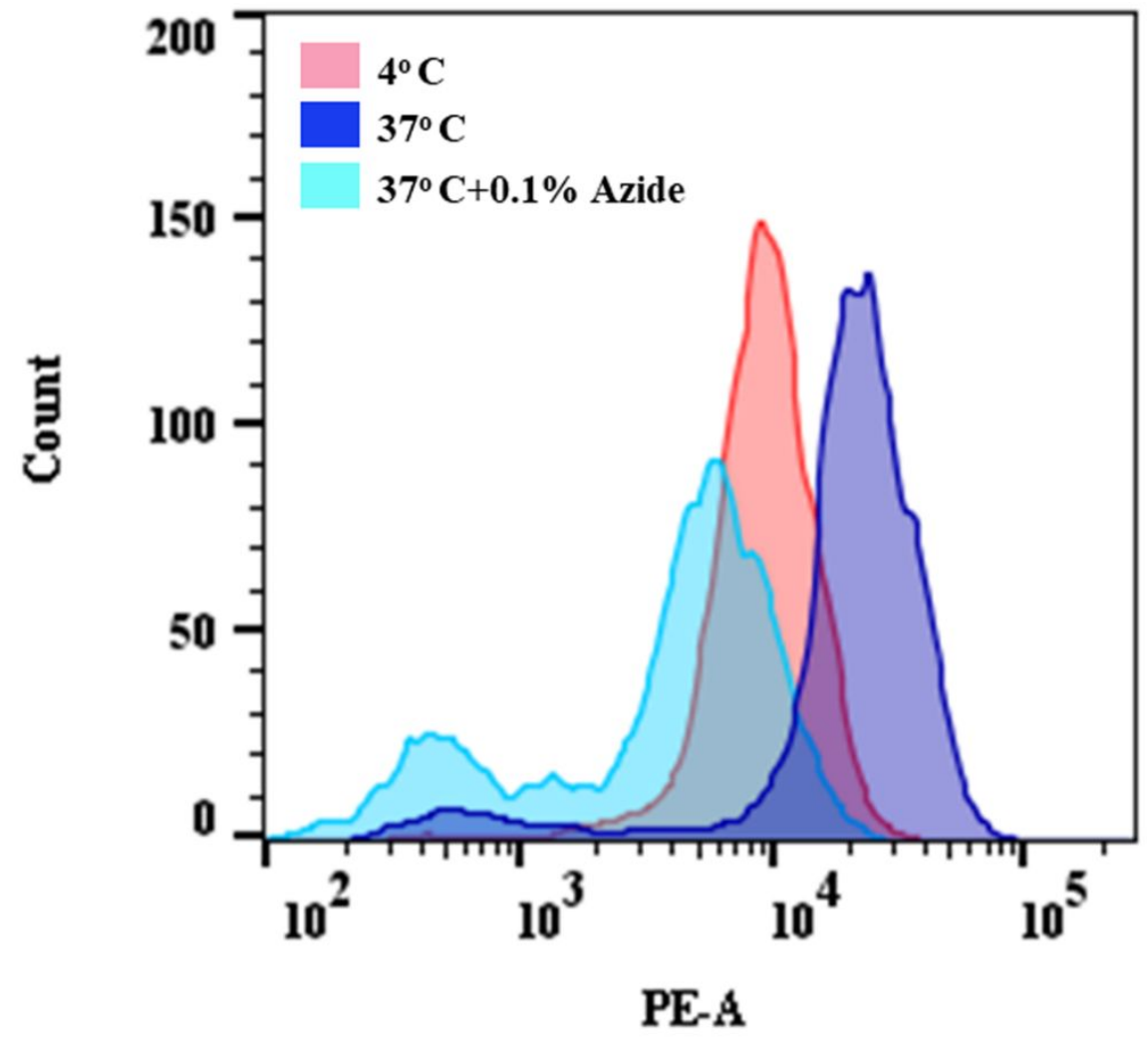

Figure S2 Representative FACS data to show uptake of fluorescent CPT particles by B16F10 cells in 1 hour under different treatment conditions including $4^{\circ} \mathrm{C}, 37^{\circ} \mathrm{C}$, and $37^{\circ} \mathrm{C}$ with cells pre-treated with $0.1 \%$ azide. 